\title{
Les mobilités : quels enjeux pour la didactique des langues et des cultures?
}

\section{Nathalie Auger}

\section{(2) OpenEdition}

1 Journals

Édition électronique

URL : http://journals.openedition.org/rdlc/6542

DOI : $10.4000 /$ rdlc.6542

ISSN : 1958-5772

Éditeur

ACEDLE

Référence électronique

Nathalie Auger, «Les mobilités : quels enjeux pour la didactique des langues et des cultures ?», Recherches en didactique des langues et des cultures [En ligne], 16-2 | 2019, mis en ligne le 30 août 2019, consulté le 25 septembre 2020. URL : http://journals.openedition.org/rdlc/6542 ; DOI : https:// doi.org/10.4000/rdlc.6542

\section{(c)}

Recherches en didactique des langues et des cultures is licensed under a Creative Commons AttributionNonCommercial-NoDerivatives 4.0 International License 
Nathalie Auger

Les mobilités : quels enjeux pour la didactique des langues et des cultures?

Ce numéro de revue fait suite à une journée d'études de l'Association des chercheurs et enseignants didacticiens des langues étrangères (Acedle) intitulée Notions en questions en didactiques des langues et qui portait sur le thème les mobilités. Cette journée d'étude, organisée le 26 janvier 2018 conjointement par l'Acedle et l'équipe Parole et Discours : fonctionnement/dysfonctionnement et appropriation de Praxiling de l'Université Paul-Valéry Montpellier 3 a exploré la notion de mobilités dans ses relations à la didactique des langues étrangères, domaine des sciences du langage, à la croisée de domaine de recherche connexes.

Cette exploration s'est consacrée aux enjeux liés aux catégorisations des mobilités et à leur pertinence pour la didactique des langues et des cultures (dorénavant DDLC). On s'est intéressé notamment aux statuts des personnes en mobilité (migrants, réfugiés, mineurs isolés, étudiants en mobilité, etc.) et aux éléments qui ont été retenus comme signifiants pour la DDLC (contextes d'enseignement, profils d'apprentissage, par exemple). Ces apports ont permis d'interroger les besoins et les projets des apprenants sur le plan de l'appropriation des langues, mais aussi les compétences liées aux mobilités et à leur valorisation. Face à ces mobilités, les enseignants également opèrent des déplacements physiques, symboliques et pédagogiques pour enseigner les langues et rencontrer leurs publics divers. A ainsi été posée la question des compétences à développer chez les enseignants de langue(s) pour faire face à / tirer parti de ces mobilités (les leurs et celles de leurs apprenants), et par conséquent, celle des modalités de formation de ces (futurs) enseignants.

Inscrit dans la tradition des Notions en Question, ce numéro s'organise autour de l'intervention d'un chercheur spécialiste du domaine à laquelle un autre chercheur réagit brièvement, à partir de ses propres travaux et réflexions. Les thématiques abordées ont été les suivantes :

\section{Enjeux des catégorisations et appropriation des langues}

Les enjeux de catégorisations sont très importants pour les apprenants et les enseignants, façons de percevoir l'autre d'une façon plus ou moins réductrice. La notion de mobilité, au regard d'autres notions telles que celles de migration par exemple, permet une décentration pertinente. En effet, si les approches sociologiques des processus de mobilités renseignent sur les critères et les modalités explicatives de ces déplacements et proposent des typologies des mobilités, elles constituent surtout pour la DDLC un appui pour réfléchir et définir des critères pertinents pour les enseignementsapprentissages des/en langues. Par ailleurs, le statut de la mobilité (entrante, sortante, volontaire, forcée, organisée, spontanée, etc.) des apprenants est devenu signifiant pour la DDLC, notamment sous la forme de didactiques dites spécialisées - langue étrangère/seconde/sur objectif spécifique/d'inclusion/de scolarisation - qui méritent d'être réinterrogées quant aux modalités d'appropriation des langues. Par exemple, le répertoire plurilingue des apprenants implique-t-il une appropriation "plurielle" des langues? Quels besoins / projets identifier chez les apprenants mobiles, entre représentations et expériences de la diversité ?

\section{Enjeux de reconnaissance et de valorisation des mobilités}

L'identification et la valorisation de compétences envisagées comme propres à la mobilité, mais aussi, inversement, la dévalorisation de l'immobilité et de comportements sédentaires sont à questionner. C'est dans ce cadre qu'ont été évoqués : 
- les conditions de possibilité et les modalités d'articulation des besoins des apprenants et des enseignants avec d'une part différents outils-cadres utilisés actuellement en DDLC (CECRL, référentiels, portfolios, etc.), notamment en matière d'évaluation et, d'autre part des programmes de mobilités, physiques ou virtuelles, proposés aux enseignants et apprenants de langues,

- les niveaux et contenus de formation à identifier, la prise en compte des imaginaires, des émotions, des dimensions sensibles dans ces processus (réflexion collaborative, auto/hétéro analyse des pratiques)

- les mobilités symboliques (réflexivité, rapport à l'altérité, trans-formation) et les moyens (décentration, journaux de bord, biographie langagière) utilisés pour y parvenir ;

- les aspects numériques et leurs conséquences sur la reconnaissance ou pas des compétences déjà-là et en devenir des apprenants.

\section{Présentation des contributions}

Le premier texte, du sociologue Eric Soriano, s'intéresse aux enjeux existant en terme de mobilité réelle ou imaginée, en partant de l'exemple des élèves arabophones en France. Ces élèves sont-ils perçus comme des migrants en potentielle difficulté scolaire ? Vivent-ils une impossible mobilité linguistique, géographique et sociale en raison des cartes scolaires ? De son point de vue, "la langue relève souvent d'un "point aveugle" dans les enquêtes en sciences sociales". Ces enquêtes permettent néanmoins de "montrer par quels mécanismes une langue peut acquérir un statut et comment ce statut peut avoir des effets pratiques sur des devenirs scolaires " où les questions de mobilités de différents ordres éclairent les pratiques et les représentations sociales liés aux langue de l'école. Jean-Louis Chiss répond à ce texte en plaidant pour " une approche linguistique et historique dans les débats sur l'immigration ". "La 'crise des migrants' est aussi une crise de mots " (Birnbaum ${ }^{1}$ 2015), et Jean-Louis Chiss montre combien les enjeux de catégorisations et de conflit de valeurs autour des langues sont « nourris de représentations parfois sans relation avec la pratique réelle de ces langues. "

Déborah Meunier, de son côté, développe une réflexion pour "penser les modalités d'une appropriation plurielle des langues et des expériences de mobilités". Son article explore les conditions de l'appropriation des langues en proposant une mise en perspective de la notion d'apprentissage. Elle se concentre sur les contextes migratoires, en élargissant à la question de la mobilité en général. Son objectif est de montrer que la posture appropriative plurielle des langues qu'elle développe permet d'interroger les dispositifs de formations, les catégories circulantes en didactique des langues et des cultures pour mettre au jour les dynamiques inclusives/ségrégatives selon les types de mobilités. Jérémi Sauvage réagit à ce texte en déplaçant le propos vers l'accueil d'étudiants en mobilité encadrée (type Erasmus) dans une université francophone. La centration sur ce type de public lui permet de revenir sur la question de ce qu'il nomme "l'accompagnement linguistique en français langue étrangère ". II revisite la catégorie Français sur Objectif Universitaire pour expliquer que les dynamiques à l'œuvre entre processus de mobilité et moyens d'appropriation de la langue étrangère ne vont pas de soi, mais résultent de rapports complexes entre l'apprenant et son contexte de mobilité.

Mathilde Anquetil présente un texte dont l'objectif est de réfléchir à la "valorisation de compétences envisagées comme propres à la mobilité, mais aussi, inversement, à la dévalorisation de l'immobilité et de comportements sédentaires". Dans un second temps, elle propose de comprendre dans quelle mesure il est possible d'articuler les besoins des apprenants et des enseignants avec les outils-cadres utilisés actuellement en DDLC (CECRL, référentiels, portfolios, etc.), notamment en matière d'évaluation. Elle propose de faire dialoguer ces deux questions en plaidant,

1 https://www.lemonde.fr/decryptages/article/2015/09/15/la-crise-des-migrants-est-aussi-une-crise-desmots_4757828_1668393.html 
tout comme Jean-Louis Chiss, pour une approche historicisée sur quatre périodes des outils de l'ingénierie éducative depuis le Processus de Bologne. Dans le même texte, Martine Derivry formule une réponse à certaines impasses pédagogiques sous la forme de pratiques possibles d'ensiegnement en langues et cultures, telles que les collaborations et télécollaborations interculturelles. Ces dispositifs de mobilité qu'elle qualifie "d'intégrés dans un paradigme plurilingue et pluriculturel " sont autant d'exemples inspirants possibles pour les enseignants et les apprenants de langues et en langues.

Contribuant à cette perspective, Marisa Cavali et Mirjam Egli proposent une réflexion à partir de la présentation d'un modèle conceptuel qu'elles inscrivent dans l'interdisciplinarité, et d'un dispositif de médiation permettant des mobilités à des fins d'apprentissage. Pour elles, la notion de médiation est au cœur des enjeux, aussi bien en ce qui concerne le processus de mobilité lui-même, que pour "l'entrée dans de nouveaux groupes ou communautés". Dans une perspective constructiviste, elles abordent les "perceptions altéritaires nécessaires" aux apprentissages. Nathalie Auger répond à cette proposition en s'appuyant sur le cas concrets d'enfants migrants arrivant en France. Cet exemple permet de montrer l'importance des processus induits par les dispositifs proposés. Finalement, les sous-bassements théoriques (socio-constructivisme) sont certainement primordiaux dans la formation des enseignants comme les auteurs l'affirment. Une perspective épistémologique est ainsi primordiale en formation. II semble également nécessaire de s'attacher aux modalités de mises en œuvre de ces concepts sous LA forme de pratiques en salle de classe en prenant en compte les mobilités à la fois physiques et symboliques des apprenants.

Ces textes issus de la journée Notions en question sont complétés, dans ce numéro, par deux textes issus de la journée d'étude Trajectoires et expériences des étudiants en mobilité, qui a eu lieu le 7 novembre 2018 à l'Université Paul-Valéry Montpellier 3. Cette journée et les articles proposés traitent du numérique en relation avec la notion de mobilité. S'ils ne se répondent pas, ils ont l'intérêt de se compléter. Jérémi Sauvage traite des Humanités numériques en lien avec une formation innovante hybride en didactique du FLE et explique en quoi ce dispositif peut présenter un intérêt majeur pour une mobilité singularisée selon les apprenants (apprentissage en autonomie et à distance). L'auteur revient sur le terme d'innovation, " tant sur le plan pédagogique que sur les plans administratif et technologique pour répondre aux besoins de mobilité actuelle des étudiants internationaux ». Quant à Jean-François Grassin, qui « interroge la figure de l'étudiant international dans une ère de la globalisation à travers le concept de mobilité, conçu comme une cinétique sociale ", il développe également une réflexion sur "les agencements matériels, relationnels et culturels que les étudiants en séjour d'étude échafaudent localement pour aboutir leur projet et apprendre ". L'analyse des traces de ces activités donne des indices de l'engagement de l'apprenant dans son projet d'étude qui peuvent permettre de construire, tout comme dans le texte de Jérémi Sauvage, des dispositifs personnalisés selon le contexte de mobilité de chacun. 